\title{
ON A VARIATIONAL PROPERTY OF INTEGRAL FUNCTIONALS AND RELATED CONJECTURES
}

\author{
BIAGIO RICCERI \\ Department of Mathematics, University of Catania \\ Viale A. Doria 6, 95125 Catania, Italy
}

The aim of this paper is to give the proofs of those results that in [4] were only announced, and, at the same time, to propose some possible developments, indicating some of the most significant open problems.

We first fix some notation. Throughout the sequel, $(T, \mathcal{F}, \mu)$ is a $\sigma$-finite non-atomic measure space, $(E,\|\cdot\|)$ is a real Banach space, whose Borel family is denoted by $\mathcal{B}(E)$, and $p$ is a real number in $[1,+\infty[$.

For simplicity, we denote by $X$ the usual space $L^{p}(T, E)$ of (equivalence classes of) strongly measurable functions $u: T \rightarrow E$ such that $\int_{T}\|u(t)\|^{p} d \mu<+\infty$, equipped with the norm $\|u\|_{X}=\left(\int_{T}\|u(t)\|^{p} d \mu\right)^{1 / p} . X^{*}$ will denote the topological dual of $X$.

Moreover, we denote by $\mathcal{A}(T \times E)$ the set of all functions $f: T \times E \rightarrow \mathbf{R}$ such that, for each $u \in X$, the function $t \rightarrow f(t, u(t))$ belongs to $L^{1}(T)$. If $f \in \mathcal{A}(T \times E)$, we put

$$
\Phi_{f}(u)=\int_{T} f(t, u(t)) d \mu
$$

for all $u \in X$.

We denote by $\mathcal{E}(T \times E)$ the set of all $\mathcal{F} \otimes \mathcal{B}(E)$-measurable functions $f: T \times E \rightarrow \mathbf{R}$ which are upper semicontinuous in $x(x \in E)$.

We denote by $\mathcal{G}(T \times E)$ the set of all functions $f: T \times E \rightarrow \mathbf{R}$ for which there are $\left.\alpha \in L^{1}(T), \gamma_{i} \in\right] 0,1\left[\right.$ and $\beta_{i} \in L^{p /\left(p-\gamma_{i}\right)}(T)(i=1, \ldots, k)$ such that

$$
-\alpha(t) \leq f(t, x) \leq \alpha(t)+\sum_{i=1}^{k} \beta_{i}(t)\|x\|^{\gamma_{i}}
$$

for almost every $t \in T$ and for every $x \in E$.

We denote by $\mathcal{V}(X)$ the family of all sets $V \subseteq X$ such that

$$
V=\left\{u \in X \mid \Psi(u)=\Phi_{g}(u)\right\}
$$

1991 Mathematics Subject Classification: Primary 49Q15; Secondary 34H05.

The paper is in final form and no version of it will be published elsewhere. 
where $\Psi \in X^{*}, g \in \mathcal{A}(T \times E)$ and $\Phi_{g}$ is Lipschitzian on $X$, with Lipschitz constant strictly smaller than $\|\Psi\|_{X^{*}}$.

Finally, we denote by $\mathcal{I}(T \times E)$ the set of all functions $f \in \mathcal{A}(T \times E)$ such that

$$
-\infty<\inf _{u \in X} \Phi_{f}(u)=\inf _{u \in V} \Phi_{f}(u)
$$

for every $V \in \mathcal{V}(X)$.

The main result of [4] was the following

Theorem 1 ([4], Theorem 1). Let $E$ be separable and $\mu$ be complete. Then, one has

$$
\mathcal{E}(T \times E) \cap \mathcal{G}(T \times E) \subseteq \mathcal{I}(T \times E) .
$$

We now prove

Theorem 2 ([4], Theorem 3). Let $\gamma \in] 0,1\left[, v \in L^{\gamma}(T, E)\right.$ and $\beta \in L^{p \gamma /(p-\gamma)}(T)$. Then, for every $V \in \mathcal{V}(X)$, one has

$$
\inf _{u \in V} \int_{T}\|v(t)-\beta(t) u(t)\|^{\gamma} d \mu=\int_{\beta^{-1}(0)}\|v(t)\|^{\gamma} d \mu .
$$

Proof. For each $(t, x) \in T \times E$, put

$$
f(t, x)=\|v(t)-\beta(t) x\|^{\gamma} .
$$

It is readily seen that the function $f$ belongs to $\mathcal{E}(T \times E) \cap \mathcal{G}(T \times E)$, and that the functional $\Phi_{f}$ is Hölder continuous in $X$, with exponent $\gamma$. So, by Remark 3 of [4] and Theorem $1, f$ belongs to $\mathcal{I}(T \times E)$. Therefore, it remains to show that

$$
\inf _{X} \Phi_{f}=\int_{\beta^{-1}(0)}\|v(t)\|^{\gamma} d \mu .
$$

To this end, fix $\varepsilon>0$ and choose $\delta>0, L \in \mathcal{F}$, with $\mu(L)<+\infty$, so that

$$
\int_{S}\|v(t)\|^{\gamma} d \mu<\varepsilon
$$

for every $S \in \mathcal{F}$ satisfying $\mu(S \cap L)<\delta$. Next, pick $n \in \mathbf{N}$ in such a way that

$$
\mu\left(L \backslash\left(\beta^{-1}(0) \cup B\right)\right)<\delta,
$$

where

$$
B=\left\{t \in L|| \beta(t) \mid \geq \frac{1}{n}\right\} \cap\{t \in L \mid\|v(t)\| \leq n\} .
$$

Finally, define $u: T \rightarrow E$ by putting

$$
u(t)= \begin{cases}\frac{v(t)}{\beta(t)} & \text { if } t \in B, \\ 0 & \text { if } t \in T \backslash B .\end{cases}
$$

Clearly, $u \in X$ and

$$
\Phi_{f}(u)<\int_{\beta^{-1}(0)}\|v(t)\|^{\gamma} d \mu+\varepsilon
$$

as desired. 
Rem ark 1. Theorem 2 tells us, in particular, that, when $\gamma \in] 0,1[$ and $\mu(T)<+\infty$, each member of the family $\mathcal{V}(X)$ is dense in $L^{\gamma}(T, E)$, equipped with the usual metric. From this, in turn, we re-obtain at once the well-known fact that the topological dual of $L^{\gamma}(T, E)$ reduces to zero. Indeed, let $\Psi$ be a continuous linear functional on $L^{\gamma}(T, E)$. Then, the restriction of $\Psi$ to $X$ belongs to $X^{*}$, and so the set $V=\{u \in X \mid \Psi(u)=0\}$ belongs to $\mathcal{V}(X)$. Consequently, owing to what above, $V$ is dense in $L^{\gamma}(T, E)$, and hence, by continuity, $\Psi$ vanishes identically there.

We now prove

Theorem 3 ([4], Theorem 4). Let $E$ be separable and let $f: T \times E \rightarrow \mathbf{R}$ be a function which is measurable with respect to $t$ and continuous with respect to $x(t \in T, x \in E)$. Moreover, assume that there exists some $\alpha \in L^{1}(T)$ such that

$$
|f(t, x)| \leq \alpha(t)
$$

for almost every $t \in T$ and for every $x \in E$.

Then, for every $V \in \mathcal{V}(X)$, one has

$$
\inf _{X} \Phi_{f}, \sup _{X} \Phi_{f}\left[\subseteq \Phi_{f}(V) .\right.
$$

So, in particular, for each $r \in] \inf _{X} \Phi_{f}, \sup _{X} \Phi_{f}\left[\right.$, the convex hull of the set $\Phi_{f}^{-1}(r)$ is dense in $X$.

Proof. Observe that both $f$ and $-f$ belong to $\mathcal{E}(T \times E) \cap \mathcal{G}(T \times E)$. Then, by Remark 2 of [4] and Theorem 1, for every $V \subseteq \mathcal{V}(X)$, we have $\inf _{X} \Phi_{f}=\inf _{V} \Phi_{f}$ and $\sup _{X} \Phi_{f}=\sup _{V} \Phi_{f}$. By Theorem 2 of [3], the set $V$ is a retract of $X$, and hence it is connected. From this, clearly (1) follows. The second part of our conclusion follows, of course, from a standard separation theorem for convex sets.

Before passing to another consequence of Theorem 2, we need the following two propositions:

Proposition 1. Let $E$ be separable, let $\left(Y,\|\cdot\|_{Y}\right)$ be another Banach space and let $\varphi: T \times E \rightarrow Y$ be a function which is strongly measurable with respect to $t$ and uniformly continuous with respect to $x(t \in T, x \in E)$. Moreover, assume that, for some $r \in\left[1,+\infty\left[\right.\right.$, there exists some $\alpha \in L^{1}(T)$ such that

$$
\|\varphi(t, x)\|_{Y}^{r} \leq \alpha(t)
$$

for almost every $t \in T$ and for every $x \in E$. Then, the Nemitski operator $N_{\varphi}: X \rightarrow$ $L^{r}(T, Y)$, defined by $N_{\varphi}(u)(t)=\varphi(t, u(t))(u \in X, t \in T)$, is uniformly continuous in $X$.

Proof. Given $\xi>0$, consider the function $\omega_{\xi}: T \rightarrow \overline{\mathbf{R}}$ defined by

$$
\omega_{\xi}(t)=\sup \left\{\delta>0 \mid\|\varphi(t, x)-\varphi(t, y)\|_{Y}^{r} \leq \xi \text { for each } x, y \in E \text { satisfying }\|x-y\|<\delta\right\} \text {. }
$$

The function $\omega_{\xi}$ is $\mu$-measurable. Indeed, given any $\lambda>0$ and chosen any countable dense set $D \subseteq E$, we readily have

$$
\left\{t \in T \mid \omega_{\xi}(t) \geq \lambda\right\}=\bigcap_{(x, y) \in D \times D,\|x-y\|<\lambda}\left\{t \in T \mid\|\varphi(t, x)-\varphi(t, y)\|_{Y}^{r} \leq \xi\right\} .
$$


Fix a set $T_{0} \in \mathcal{F}$, with $\mu\left(T \backslash T_{0}\right)=0$, such that, for each $n \in \mathbf{N}$, the restriction of $\omega_{1 / n}$ to $T_{0}$ is measurable. Now, fix $\varepsilon>0$ and $\left.\eta \in\right] 0, \varepsilon / 2^{r}\left[\right.$. Pick $\delta_{1}>0$ and $L \in \mathcal{F}$, with $\mu(L)<+\infty$, in such a way that

$$
\int_{S} \alpha(t) d \mu<\eta
$$

for every $S \in \mathcal{F}$ satisfying $\mu(S \cap L)<\delta_{1}$. Also, choose $m, n \in \mathbf{N}$ so that $m>\mu(L) /(\varepsilon-$ $\left.2^{r} \eta\right)$ and

$$
\mu\left(L \backslash\left(\omega_{\frac{1}{m}}^{-1}(] \frac{1}{n},+\infty[) \cap T_{0}\right)\right)<\frac{\delta_{1}}{2} .
$$

Finally, pick $\delta \in] 0, \delta_{1} /\left(2 n^{p}\right)\left[\right.$. Now, let $u, v \in X$ satisfy $\|u-v\|_{X}^{p}<\delta$. Put

$$
S=\left\{t \in L \cap T_{0} \mid\|u(t)-v(t)\|<\frac{1}{n}<\omega_{\frac{1}{m}}(t)\right\} .
$$

Then, taken into account that $\mu(L \backslash S)<\delta_{1}$, we have

$$
\begin{aligned}
\left\|N_{\varphi}(u)-N_{\varphi}(v)\right\|_{L^{r}(T, Y)}^{r} & =\int_{T}\|\varphi(t, u(t))-\varphi(t, v(t))\|_{Y}^{r} d \mu \\
& <2^{r} \int_{(T \backslash L) \cup(L \backslash S)} \alpha(t) d \mu+\int_{S}\|\varphi(t, u(t))-\varphi(t, v(t))\|_{Y}^{r} d \mu \\
& <2^{r} \eta+\frac{\mu(L)}{m}<\varepsilon .
\end{aligned}
$$

The proof is complete.

If $h$ is a Gâteaux differentiable real function on $E$, we denote by $h^{\prime}$ its Gâteaux derivative. We denote by $C^{1}(E)$ the space of all continuously Gâteaux differentiable real functions on $E$.

Proposition 2. Let $E$ be reflexive and separable, let $p>1$, and let $f: T \times E \rightarrow \mathbf{R}$ be a function which is $\mu$-measurable in $t(t \in T)$. Moreover, assume that, for some $\psi \in L^{\frac{p}{p-1}}(T)$ and for almost every $t \in T, f(t, \cdot) \in C^{1}(E), f_{x}^{\prime}(t, \cdot)$ is uniformly continuous in $E$ and

$$
\sup _{x \in E}\left\|f_{x}^{\prime}(t, x)\right\|_{E^{*}} \leq \psi(t) .
$$

Then, the functional $\Phi_{f}$ is Gâteaux differentiable in $X, \Phi_{f}^{\prime}$ is uniformly continuous, and one has $f_{x}^{\prime}(\cdot, u(\cdot)) \in L^{p /(p-1)}\left(T, E^{*}\right)$ and

$$
\left\langle\Phi_{f}^{\prime}(u), v\right\rangle=\int_{T}\left\langle f_{x}^{\prime}(t, u(t)), v(t)\right\rangle d \mu
$$

for all $u, v \in X$. Furthermore, one has

$$
\left\|\Phi_{f}^{\prime}(u)\right\|_{X^{*}}=\left(\int_{T}\left\|f_{x}^{\prime}(t, u(t))\right\|_{E^{*}}^{\frac{p}{p-1}} d \mu\right)^{\frac{p-1}{p}}
$$

for all $u \in X$.

Proof. Let $u, v \in X$ be fixed. Choose any sequence $\left\{\lambda_{n}\right\}$ in $\mathbf{R} \backslash\{0\}$ converging to zero. For each $n \in \mathbf{N}, t \in T$, put

$$
h_{n}(t)=\frac{f\left(t, u(t)+\lambda_{n} v(t)\right)-f(t, u(t))}{\lambda_{n}} .
$$


Clearly, each function $h_{n}$ is $\mu$-measurable, and, for almost every $t \in T$, the sequence $\left\{h_{n}(t)\right\}$ converges to $\left\langle f_{x}^{\prime}(t, u(t)), v(t)\right\rangle$. On the other hand, thanks to the mean-value theorem, we have

$$
\sup _{n \in \mathbf{N}}\left|h_{n}(t)\right| \leq \psi(t)\|v(t)\|
$$

a.e. in $T$. Then, by the dominated convergence theorem, we get

$$
\lim _{n \rightarrow \infty} \int_{T} h_{n}(t) d \mu=\int_{T}\left\langle f_{x}^{\prime}(t, u(t)), v(t)\right\rangle d \mu .
$$

Of course, this shows $(2)$. The fact that $f_{x}^{\prime}(\cdot, u(\cdot))$ belongs to $L^{p /(p-1)}\left(T, E^{*}\right)$ follows from the nature of $E$ (take into account that also $E^{*}$ is reflexive and separable) via Pettis's measurability theorem ([2], p. 42). The validity of (3) follows from standard reasonings (see, for instance, [2], pp. 97-98). Finally, the uniform continuity of the operator $\Phi_{f}^{\prime}$ is an easy consequence of Proposition 1, applied taking $Y=E^{*}$ and $\varphi=f_{x}^{\prime}$.

Now, we can prove

TheOREm 4 ([4], Theorem 5). Let $E$ be reflexive and separable, let $p>1$, and let $f \in \mathcal{E}(T \times E) \cap \mathcal{G}(T \times E)$. Moreover, assume that, for some $\psi \in L^{p /(p-1)}(T)$ and for almost every $t \in T, f(t, \cdot) \in C^{1}(E), f_{x}^{\prime}(t, \cdot)$ is uniformly continuous in $E$ and

$$
\sup _{x \in E}\left\|f_{x}^{\prime}(t, x)\right\|_{E^{*}} \leq \psi(t) \text {. }
$$

Then, for every $V \in \mathcal{V}(X)$, there exists a sequence $\left\{u_{n}\right\}$ in $V$ such that

$$
\lim _{n \rightarrow \infty} \Phi_{f}\left(u_{n}\right)=\inf _{u \in X} \Phi_{f}(u)
$$

and

$$
\lim _{n \rightarrow \infty} \int_{T}\left\|f_{x}^{\prime}\left(t, u_{n}(t)\right)\right\|_{E^{*}}^{\frac{p}{p-1}} d \mu=0 .
$$

Proof. Let $V \in \mathcal{V}(X)$. By Remark 2 of [4] and Theorem 1, there exists a sequence $\left\{u_{n}\right\}$ in $V$ such that

$$
\lim _{n \rightarrow \infty} \Phi_{f}\left(u_{n}\right)=\inf _{X} \Phi_{f} .
$$

According to a consequence of Ekeland's variational principle ([1], p. 259), we can then find a sequence $\left\{v_{n}\right\}$ in $X$ such that

$$
\lim _{n \rightarrow \infty}\left\|u_{n}-v_{n}\right\|_{X}=0
$$

and

$$
\lim _{n \rightarrow \infty}\left\|\Phi_{f}^{\prime}\left(v_{n}\right)\right\|_{X^{*}}=0 .
$$

By Proposition 2, $\Phi_{f}^{\prime}$ is uniformly continuous, and hence we have

$$
\lim _{n \rightarrow \infty}\left\|\Phi_{f}^{\prime}\left(u_{n}\right)\right\|_{X^{*}}=0
$$

An appeal to (3) completes the proof.

To conclude, we now state, under the form of conjectures, the open problems to which we alluded at the beginning of the paper. We assume that $E$ and $\mu$ are as in Theorem 1 . 
Conjecture 1. Let $U \subseteq X$ be closed and such that $\inf _{X} \Phi_{f}=\inf _{U} \Phi_{f}$ for every $f \in \mathcal{E}(T \times E) \cap \mathcal{G}(T \times E)$. Then, there exists some $V \in \mathcal{V}(X)$ such that $V \subseteq U$.

Conjecture 2. There exists some function $f: T \times E \rightarrow \mathbf{R}$ such that $f \in \mathcal{G}(T \times E) \backslash$ $\mathcal{I}(T \times E)$ and $-f \in \mathcal{E}(T \times E)$.

\section{References}

[1] J.-P. Aubin and I. Ekeland, Applied Nonlinear Analysis, John Wiley \& Sons, 1984.

[2] J. Diestel and J. J. Uhl, Jr., Vector measures, Math. Surveys 15, Amer. Math. Soc., 1977.

[3] B. Ricceri, Structure, approximation et dépendance continue des solutions de certaines équations non linéaires, C. R. Acad. Sci. Paris, Série I, 305 (1987), 45-47.

[4] B. Ricceri, A variational property of integral functionals on $L^{p}$-spaces of vector-valued functions, C. R. Acad. Sci. Paris, Série I, 318 (1994), 337-342. 